\title{
Future powertrain concepts for sustainable mobility
}

Dr. Werner Willems, Ford Forschungszentrum Aachen GmbH

Die Unterlagen wurden nicht zur Veröffentlichung freigegeben.

Wir bitten um Verständnis. 